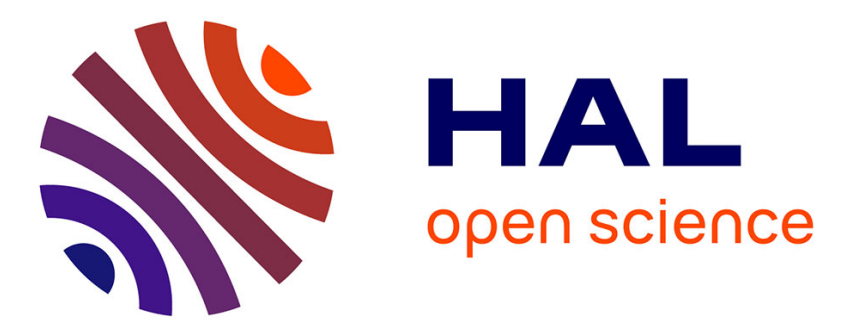

\title{
L'enseignement littéraire à l'école. Quand la transmission du goût pour la lecture cède le pas à la valorisation du patrimoine littéraire
}

Emmanuelle Guittet

\section{- To cite this version:}

Emmanuelle Guittet. L'enseignement littéraire à l'école. Quand la transmission du goût pour la lecture cède le pas à la valorisation du patrimoine littéraire. Culture et Musées, 2021, 38, pp.73-97. 10.4000/culturemusees.6712 . hal-03471482

\section{HAL Id: hal-03471482 \\ https://hal.science/hal-03471482}

Submitted on 8 Dec 2021

HAL is a multi-disciplinary open access archive for the deposit and dissemination of scientific research documents, whether they are published or not. The documents may come from teaching and research institutions in France or abroad, or from public or private research centers.
L'archive ouverte pluridisciplinaire HAL, est destinée au dépôt et à la diffusion de documents scientifiques de niveau recherche, publiés ou non, émanant des établissements d'enseignement et de recherche français ou étrangers, des laboratoires publics ou privés. 


\section{OpenEdition}

Journals

\section{Culture \& Musées}

Muséologie et recherches sur la culture

$38 \mid 2021$

Patrimonialisations de la littérature

Dossier

L'enseignement littéraire à

l'école. Quand la transmission du goût pour la lecture cède le pas à la valorisation du patrimoine littéraire

Literary education in schools. When transmitting taste gives way to highlighting literary heritage

La educación literaria en la escuela. Cuando la transmisión del gusto por la lectura da paso a la valorización del patrimonio literario

EMMANUELLE GUITTET

p. $73-97$

https://doi.org/10.4000/culturemusees.6712

\section{Résumés}

Français English Español

Dans cet article, nous interrogeons les effets de l'enseignement littéraire à l'école sur les représentations et pratiques de lecture. S’il peine à opérer une médiation au livre et à la lecture efficace, il n'est néanmoins pas sans effets puisqu'il participe à la patrimonialisation d'œuvres littéraires, par leur sélection dans les programmes et par leur analyse dans le cadre des cours de français. Cette patrimonialisation d'un corpus littéraire n'est à son tour pas sans effets sur les représentations associées par les lecteurs et lectrices à cette littérature (entre rejet, sacralisation et intimidation), qui s'accompagnent de pratiques de lectures ambivalentes et socialement situées (peu lisent des classiques mais tous et toutes reconnaissent la supériorité de cette littérature). Que la littérature de classiques suscite le rejet, qu'elle soit intégrée à un ensemble de lectures sous la forme d'obligation, ou qu'elle corresponde à une réelle appétence, force est de constater que l’institution scolaire - par le biais des lectures imposées - parvient à asseoir la légitimité de cette littérature.

In this article, we propose an examination of the effects of literary education in schools on the representations and practices of reading. While schools seem to struggle to offer an effective intermediary to books and reading, they are nonetheless not without importance because they participle in the patrimonialisation of certain books by including them in curricula and analyzing 
them in language classes. This process as regards a literary corpus is not without effect on the representations of readers of this literature (be it rejection, sacralization or intimidation), that are accompanied by reading practices both ambivalent (few read the classics but the superiority of such texts is universally recognized) and socially situated. That classic literature should elicit rejection, or be incorporated into obligatory reading programs, or correspond to a true taste, one is obliged to recognize that the education system, through its system of required reading, contributes to the legitimacy of such literature.

En este artículo se examinan los efectos de la educación literaria en la escuela en torno a las representaciones y prácticas de la lectura. Pese a la dificultad de efectuar una mediación entre el libro y la lectura eficaz, no por ello carece de efectos, ya que participa en la patrimonialización de ciertas obras literarias, a través de su selección en los programas educativos y su análisis en el marco de los cursos de francés. Esta patrimonialización de un corpus literario no está a su vez exenta de efectos sobre las representaciones de los lectores y las lectoras sobre esta literatura (entre rechazo, sacralización e intimidación), que se acompañan de prácticas de lectura ambivalentes (pocos leen clásicos, pero todos reconocen la superioridad de esta literatura) y socialmente situadas. Que la literatura de los clásicos suscite rechazo, que se integre en un programa de lecturas obligatorias, o que corresponda a una apetencia real, está claro que la institución escolar -mediante las lecturas obligatorias- logra establecer la legitimidad de esta literatura.

\section{Entrées d'index}

Mots-clés : œuvres classiques, institution scolaire, pratiques de lecture, lectures imposées, patrimoine littéraire, lecture esthète

Keywords: classics, schools, reading practices, required reading, literary heritage, taste for reading

Palabras clave: obras clásicas, institución escolar, prácticas de lectura, lectura obligatoria, patrimonio literario, lectura estética

\section{Notes de la rédaction}

Manuscrit reçu le 8 janvier 2021

Version révisée reçue le 15 mai 2021

Article accepté pour publication le 26 juin 2021

\section{Texte intégral}

La lecture fait l'objet, depuis le milieu du xxe siècle, d'un ensemble de discours institutionnels émanant de l'État, de l'Église ou encore de l'école, et est devenue dans les années 1950 " une pratique culturelle dans la hiérarchie des valeurs » (Chartier \& Hébrard, 2000). Ces discours marquent une volonté de développer la lecture publique et s'accompagnent des premières enquêtes quantitatives portant sur la lecture, ainsi que de l'émergence de politiques culturelles liées à la démocratisation de la culture (Hersent, 2000) et d'un intérêt croissant pour le patrimoine dans les politiques publiques (Poulot, 2006).

2 Ces enquêtes pionnières (Dumazedier \& Hassenforder, $1959^{1}$; Guillemot et al., 1963) témoignent de la volonté publique de mieux comprendre les différentes manières dont les pratiques de lecture se déploient (ou non) dans la population, ainsi que les freins à leur développement. Elles mettent notamment en avant des modalités de lecture et des difficultés spécifiques rencontrées par les lecteurs et lectrices des classes populaires. Les résultats de ces enquêtes poussent malgré tout leurs auteurs et autrices à se montrer optimistes quant à l'avenir des pratiques de lecture (Hersent, 2000) : la généralisation de la scolarisation secondaire et supérieure ainsi que l'augmentation des niveaux de vie éveillent en effet l'espoir d'une diminution des écarts entre les classes sociales vis-à-vis de l'accès à la lecture. Mais les enquêtes menées au fil des soixante années nous séparant de ces premières études n'ont pas démontré que les écarts entre les différentes couches de la population s'étaient estompés, qu'il s'agisse des inégalités scolaires ou de celles relatives à l'accès au livre (Bourdieu \& Passeron, 1970 ; Lahire, 1993a, 1993b ; Donnat, 2009; Lombardo \& Wolff, 2020).

La fin des années 1980 a également été marquée par l'apparition de nouvelles inquiétudes vis-à-vis d'une baisse de la lecture constatée dans l'ensemble de la 
population. L'enquête menée par Baudelot et ses collègues sur les lectures des collégiens en témoigne notamment. Selon les auteurs, le « discours contemporain sur la lecture comporte deux éléments : une valorisation inconditionnelle de la lecture et une inquiétude sociale pour les non-lecteurs, dont on déplore l'accroissement » (Baudelot et al., 1999 : 11), ce qui est associé à la baisse du niveau scolaire. Le poids des espoirs investis dans le développement de la lecture ainsi que les bienfaits qui lui sont communément associés ${ }^{2}$ sont en effet au fondement des incitations multiples à lire - de la part d'un ensemble varié d'institutions.

4 Dans ce contexte, l'enseignement du français au collège et au lycée apparait comme un cadre potentiel de médiation au livre de grande ampleur, qui touche une très vaste partie de la population. Les lectures imposées sont par ailleurs bien souvent l'occasion d'une rencontre initiale ou nouvelle avec le livre, et les cours de français sont également le cadre d'une prescription scolaire. Il s'agit dans cet article d'étudier l'influence de cette prescription spécifique sur le développement des pratiques de lecture et sur la formation des goûts des lecteurs et lectrices. Cette question nous amènera à penser la découverte scolaire des textes littéraires non comme une forme de médiation au livre et à la lecture efficace, mais comme un outil de patrimonialisation de la littérature - ou, du moins, d'une certaine littérature.

$5 \quad$ S'il est possible de définir le classique par son caractère universel et par la durée de sa réputation (Becker, 2010 : 361), on sait le caractère construit de cette catégorie d'œuvres. Selon Alain Viala (1993 : 25), le processus de classicisation est formé par " toute une série de manipulations qui font les classiques ». Les premières étapes de ce processus s'appuient sur l'ensemble des mécanismes de prescription (critique, prix littéraires, succès de librairie, etc.), mais le processus ne s'achève que par l'inscription des œuvres dans le temps long de la patrimonialisation, ce qui distingue les œuvres classiques des nouveautés littéraires dont la notoriété repose sur l'actualité (Guittet, 2018).

6 C'est dans la constitution d'un corpus canonique d'œuvres que se lit, selon Viala (1992 : 10), " la puissance de l'institution scolaire dans la définition du classique »: l'inscription dans les programmes scolaires achève la patrimonialisation des œuvres et leur confère un statut à part, ce qui a d'autant plus d'effets que les œuvres patrimonialisées accompagnent l'apprentissage de la lecture et de l'écrit pour l'ensemble des élèves. Le fait qu'elles figurent dans les programmes, qu'elles soient lues et analysées en classe, constitue donc - pour les élèves et les adultes qu'ils deviendront - la preuve qu'une œuvre a été consacrée par le temps long de la classicisation. Ceci a des effets d'autant plus forts que "l'action pédagogique tend à produire la légitimité de ce qu'elle transmet en désignant ce qui est transmis, du seul fait de le transmettre légitimement, comme digne d'être transmis, par opposition à tout ce qu'elle ne transmet pas » (Bourdieu \& Passeron, 1970 : 37).

7 Au moment présent, les œuvres dites « classiques » n'ont donc par définition pas besoin du support de la prescription littéraire des médias ou des libraires pour que leur valeur soit reconnue auprès des publics, puisque l'école leur assure la reconnaissance et une diffusion d'ampleur qui touche la majorité des individus de chaque génération. À cet égard, l'institution scolaire est à la littérature ce que le musée est implicitement à l'art, en ce qu'ils participent tous deux à la patrimonialisation des œuvres : par la construction d'un passé commun et la transmission de ce passé. De l'enseignement littéraire, l'on pourrait retenir ce que Dominique Poulot écrit du musée, «l'un des dispositifs qui "logent" le passé dans les ressources du présent » (Appadurai, 1981; Poulot, 2011), au même titre que la bibliothèque qui, par la constitution d'un fonds d'auteurs, participe à la reconnaissance du canon littéraire (Rabot, 2012).

8 L'enseignement littéraire à l'école, et particulièrement au collège et au lycée, porte donc un ensemble d'enjeux que nous proposons de questionner dans cet article, notamment sous l'angle de la transmission d'un goût pour la lecture et de la valorisation du patrimoine littéraire. Nous montrerons notamment que si les lectures imposées en cours de français ont accompagné de façon inégale le développement de la lecture chez les adolescents, le rapport que ces derniers entretenaient avec elle n'est pas sans incidence sur leurs goûts littéraires et les œuvres sur lesquelles se portent leurs lectures une fois adultes. Quel que soit le rapport que les lecteurs et lectrices de romans 
entretiennent par la suite avec la catégorie des classiques, nous montrerons que l'école, par le biais des lectures imposées, parvient à asseoir la légitimité de cette littérature.

Cet article repose sur une enquête menée dans le cadre d'une thèse de sociologie qui avait pour objet la réception de la prescription littéraire par les lecteurs de romans. Celle-ci a été menée à travers 36 entretiens semi-directifs et 457 questionnaires auprès de lecteurs et lectrices âgés de 18 à 65 ans, résidant à Paris ou en proche banlieue, ayant lu au moins un roman au cours des douze derniers mois et actifs 3 . Bien que non spécifiquement structurée autour de la question des lectures imposées et de la prescription scolaire, elle a néanmoins permis, puisqu'il s'agissait d'étudier les pratiques de lecture dans leur ensemble, de mettre au jour l'influence de l'institution scolaire sur le développement des pratiques de lecture et de compétences des lecteurs, ainsi que sur les goûts littéraires et les choix opérés par les lecteurs au sein de l'offre éditoriale une fois adultes.

\section{Un contexte de médiation au livre difficile?}

Rappelons que dans le cadre de l'enseignement du français, les élèves de collège et lycée se trouvent face à des textes extraits d'œuvres littéraires classiques, pour la plupart publiées au xixe siècle, faisant partie d'un programme, et sur lesquels un ensemble d'exercices (notamment commentaire et dissertation) vont s'appuyer. Ces textes, appartenant au patrimoine littéraire français, existent en dehors de l'école où ils ont déjà été rencontrés par certains élèves, alors que pour d'autres, l'école sera le lieu de leur découverte.

\section{Une médiation générant peu d'effets sur le développement de la lecture}

Les données produites dans le cadre de l'enquête montrent, tant au travers des questionnaires que des entretiens menés, le peu d'effet de l'enseignement littéraire sur le développement ou le maintien d'une pratique de lecture par la suite : autant de lecteurs interrogés déclarant s'être au moins un peu intéressés aux lectures imposées au collège et au lycée présentent au moment de l'enquête une faible, moyenne ou grande pratique de lecture 4 (exactement répartis en trois tiers). La grande majorité des lecteurs et lectrices attribuent à l'enseignement littéraire un moindre rôle dans leur goût et leur pratique, ce que remarquait déjà Erich Schön (1993 : 34) lorsqu'il écrivait que " même des gens qui lisent régulièrement n'en tiennent quasiment aucun compte dans la reconstruction de leur parcours ». La plupart des personnes interrogées s'accordent, quels que soient leur trajectoire et le moteur initial de leur pratique, à affirmer que l'école n'a pas été ce moteur, voire aurait plutôt représenté un frein dans le développement de leur pratique.

12 En revanche, la corrélation entre les pratiques de lecture des membres de l'entourage familial et l'intérêt déclaré des enquêtés pour les lectures imposées est très marquée : $75 \%$ des lecteurs interrogés dont les membres de la famille lisaient beaucoup déclarent en effet s'être au moins un peu intéressés aux lectures imposées, contre $52 \%$ des lecteurs dont l'entourage familial était peu ou pas tourné vers la lecture.

Figure 1. Intérêt pour les lectures imposées en fonction de la fréquence de lecture de l'entourage familial. 


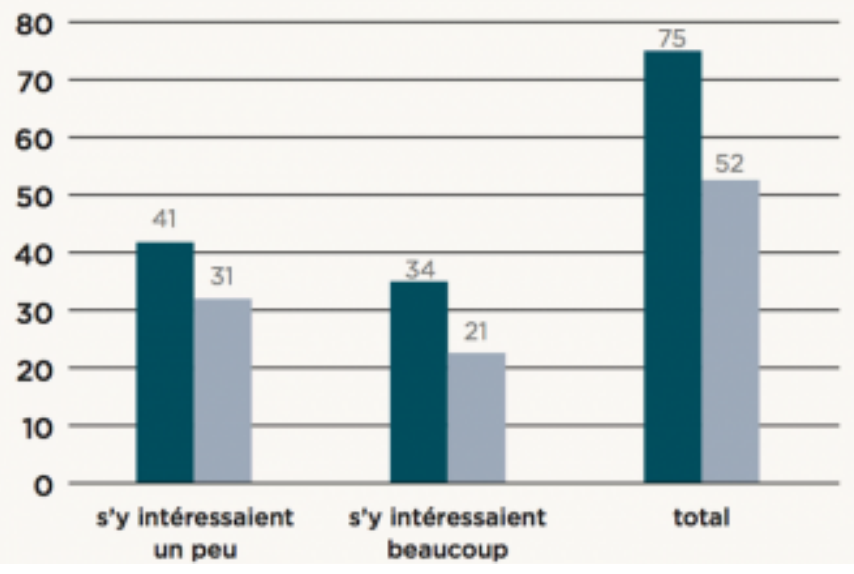

Ces données pointent l'influence des pratiques familiales de lecture sur l'intérêt pour les lectures imposées des élèves et rappellent qu'en matière d'intérêt pour la culture, tout indique que les pratiques se jouent avant et en dehors de l'école.

\section{Deux profils d'adolescents particulièrement compatibles avec l'enseignement littéraire}

Les élèves issus de familles au sein desquelles l'ambiance livresque (Singly, 1989) est favorable au développement de la lecture ont en effet plus de chance d'hériter de dispositions les poussant à développer une pratique personnelle de la lecture (Bourdieu, 1979), de même qu'un rapport au monde ainsi qu'un ensemble de compétences vis-à-vis de l'écrit rendant possible une familiarité avec l'enseignement du français (Lahire, 1993a). Ces élèves, dont les parents ont généralement une pratique de lecture, sont plus fréquemment dotés de toutes les compétences et appétences expliquant une familiarisation rapide avec l'univers scolaire, ainsi que des représentations positives associées au travail sur les textes littéraires. Ils ont d'autre part de fortes probabilités d'avoir déjà rencontré les œuvres classiques étudiées avant et en dehors de l'école. Il s'agit des individus dont le développement de la lecture comme pratique de loisirs dépend le moins de l'institution scolaire, et de ceux pour lesquels l'enseignement de la littérature à l'école est de fait (et paradoxalement) le plus accessible.

Certaines conditions (les incitations aux pratiques de la lecture et de l'écriture, le degré d'ascétisme scolaire encouragé dans la famille et le surinvestissement, le militantisme dans les familles, ou encore un rapport méticuleux au travail, quel qu'il soit) viennent expliquer chez des enfants issus des classes populaires et moyennes des rapports à l'éducation scolaire qui permettent à l'école de jouer un rôle socialisateur et transmetteur de pratiques (Lahire, 1995). Ainsi, les « tard-venus », pour reprendre l'expression de Fanny Renard, « découvrent la lecture dans le cadre d'un enseignement scolaire réclamant l'acquisition et l'adoption d'un rapport analytique au langage et aux textes » (Renard, 2011 : 49), enseignement qui implique évaluation, correction, délai de lecture imposé, etc. Le rapport de ces individus à la lecture est donc dans un premier temps teinté par l'institution scolaire et ses règles, ce qui les rend plus enclins à développer un rapport sacré à la littérature et au livre, et plus sensibles à la prescription par les enseignants (Singly, 1989). Les individus dont l'ascension sociale dépend le plus directement de l'école sont en effet amenés à faire preuve de docilité scolaire et à reconnaître la légitimité de l'institution (Bourdieu \& Passeron, 1970 : 43).

En somme, certains lecteurs et lectrices sont plus sensibles à l'enseignement littéraire à l'école : des lecteurs héritiers dont la pratique ultérieure ne découle pas des lectures scolaires et qui n'ont donc a priori pas besoin d'une médiation au livre et à la lecture, et des individus - rares dans notre échantillon (3 sur 36 enquêtés) - qui ont développé un goût pour la lecture en parallèle d'une réussite scolaire associée à une mobilité sociale. Les autres, c'est-à-dire la majorité des élèves découvrant la lecture d'œuvres littéraires 
au fil de leur parcours scolaire, sont peu disposés à adhérer à l'enseignement de la littérature au collège et au lycée.

\section{Des textes et des modalités de lecture jugés inadaptés}

Questionnées au sujet de leurs souvenirs de lectures imposées, la plupart des personnes interrogées, et ne correspondant pas à l'un des deux profils décrits plus haut, n'ont spontanément parlé que des lectures et de l'enseignement donnés au lycée ; c'est sans doute parce que les lectures imposées au lycée sont puisées dans un répertoire moins contemporain qu'au collège - malgré une forte appétence des adolescents pour la lecture d'œuvres qui leur parlent et leur parlent d'eux (Étienne, 2015) - que ces lectures ont laissé une trace indélébile dans leurs souvenirs en raison de l'expérience déplaisante qui en a découlé.

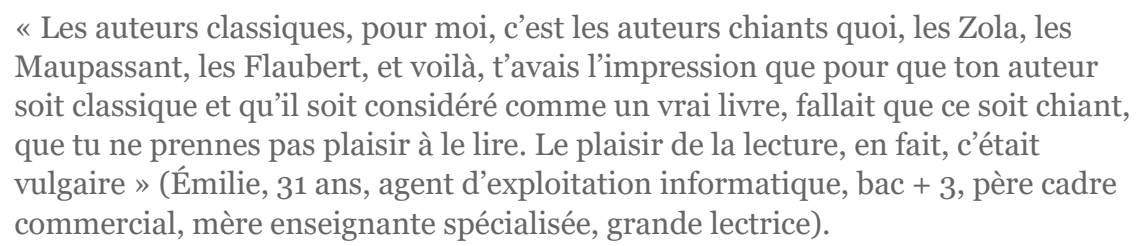

On voit toute la résistance à l'injonction scolaire à lire des classiques dans les propos tenus par cette lectrice - ayant par ailleurs construit sa pratique de la lecture en opposition à l'idée que certaines ouvres seraient plus légitimes que d'autres, et privilégiant l'évasion et l'imaginaire ainsi que des lectures de " genre ». Ici, ennui et légitimité vont de pair. Si cette lectrice fait sans doute partie des plus émancipés vis-àvis de la hiérarchisation des pratiques de lecture et des genres littéraires, on peut malgré tout lire dans ses propos la marque d'un rejet globalement répandu au sein des classes moyennes et populaires, certains lecteurs allant même jusqu'à parler de " traumatisme ». Mais ce désintérêt de la littérature classique pour son caractère ennuyeux se retrouve également chez les lecteurs appartenant aux classes supérieures, notamment parce qu'ayant déjà accès au plaisir de la lecture, ils ont pu développer une pratique s'appuyant sur d'autres types de textes littéraires ou d'autres types de rapports aux textes (fondés sur l'évasion, notamment). Ce constat rejoint ce qu'a relevé Stéphanie Kellner (2020) au sujet des emprunteurs de liseuse en bibliothèque, de même que des attitudes des bibliothécaires face au fonds classique : les œuvres " associé[e]s à la prescription scolaire et à une littérature d'un autre temps » sont mises à distance malgré la bonne volonté culturelle des usagers.

Les lecteurs ayant été interrogés une fois adultes, il est fréquent qu'ils reviennent sur les choix de textes et la manière dont ils sont enseignés, avec un regard plus critique et distancié, et ce, d'autant plus lorsqu'ils sont parents à leur tour puisque leur expérience de l'école est renouvelée à l'occasion de la scolarisation de leurs propres enfants.

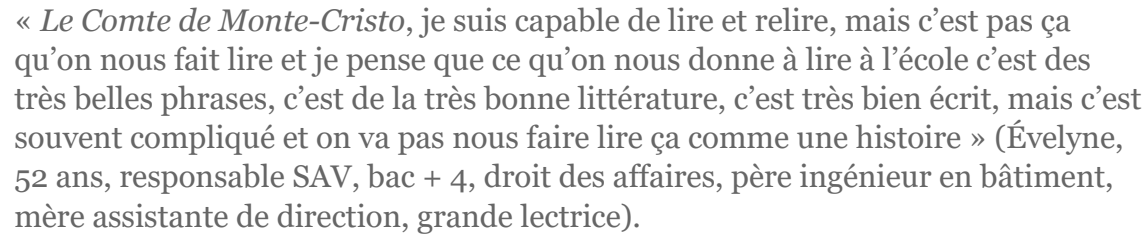

Ce discours rétroactif sur les programmes scolaires de lectures imposées est la marque d'une distanciation par rapport à la légitimité de l'école à imposer des textes, puisqu'il ne part pas d'une autocritique - comme c'est le cas pour certains lecteurs portant un regard négatif sur leurs propres compétences littéraires - mais d'une critique de l'institution. Ce qui est contesté, c'est la capacité de l'école à transmettre le plaisir de lire, et notamment en raison d'un choix d'œuvres considéré inadapté aux goûts des élèves, et de l'enseignement qui est fait de ces textes, mettant en avant l'analyse des textes au détriment du plaisir de lire. 
Plus les adolescents avancent dans leur scolarité, plus les lectures servent de support à l'analyse de texte et au développement de compétences littéraires. L'enseignement littéraire au lycée est associé par les lecteurs - qui évoquent des (mauvais) souvenirs très vifs de cette période de leur vie - à un grand nombre de contraintes. Entre l'imposition de textes, le délai de lecture et le travail qui doit être réalisé à partir des textes, la contrainte représentée par les modalités de cet enseignement représente une entrave au développement d'une pratique pour le plaisir, pourtant au centre des attentes des jeunes vis-à-vis des livres (Singly, 1993 : 134).

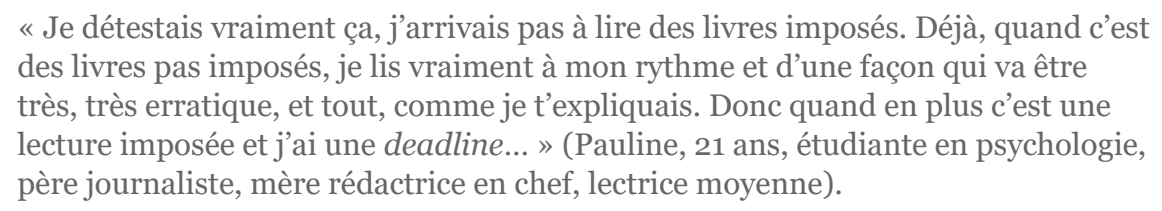

Tant du point de vue du choix des textes que de la manière dont ils sont abordés et du cadre décrit comme coercitif dans lequel ils le sont, il y a donc un décalage entre les attentes des lecteurs et les conditions de l'enseignement littéraire, principalement au lycée.

\section{La construction d'un rapport esthète et savant à la littérature}

C'est parce que, dans les imaginaires collectifs, l'école est supposée jouer un rôle de médiation au livre que son action est perçue par la majorité des personnes interrogées comme un échec. Mais si l'institution scolaire peine à transmettre un goût pour la lecture, cela ne signifie pas qu'elle n'a pas d'effets sur les pratiques de lecture ou sur les représentations associées aux œuvres littéraires par les lecteurs. Il semble en effet que ce que l'école cherche à accomplir auprès des élèves se situe plutôt autour de la transmission d'un type de rapport au texte et de compétences analytiques. Le programme de l'enseignement commun de français en classes de seconde et de première littéraire, publié au Bulletin officiel en 2010 (Éducation nationale, 2010), indique d'ailleurs, parmi les finalités du programme de français :

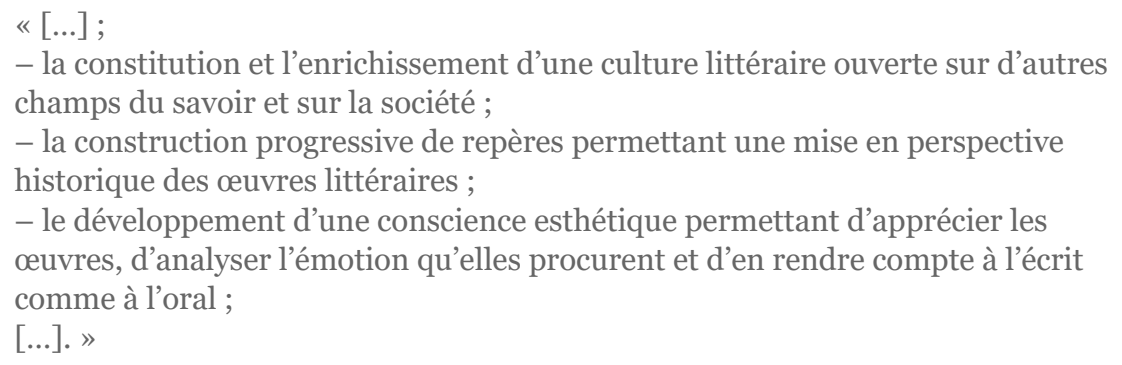

Et il stipule que la réalisation de ces objectifs doit prendre « appui principalement sur la lecture et l'étude de textes majeurs de notre patrimoine».

Morgane Maridet écrit à cet égard que, " dans cette construction d'un rapport esthétique - plutôt qu'éthico-pratique - au texte, c'est une façon de lire de classe qui s'élabore », et que le rapport aux œuvres ainsi constitué se fonde sur un déplacement de l'intérêt du contenu des œuvres vers leur forme, ainsi que « vers les effets proprement artistiques qui ne s'apprécient que relationnellement, par une comparaison avec d'autres œuvres » (Maridet, $2016: 167)$. L'enseignement du français, par la « spécificité des savoirs et savoir-faire lectoraux enseignés en classe » (Renard, $2011: 42$ ) - tels que la contextualisation, l'analyse et les mises en lien des œuvres entre elles -, contribue en effet non pas à créer des lecteurs mais à leur transmettre des compétences littéraires et des modes d'appropriation des textes particuliers. C'est également ce que remarque Florence Eloy lorsqu'elle évoque les attendus de l'enseignement de la musique au collège : plus que de pousser les élèves vers l'écoute d'un certain type d'œuvres, le 
curriculum de l'Éducation nationale encourage l'acquisition d'un « savoir-écouter », de même que l'apprentissage d'une écoute « esthète » (Eloy, 2015 : 37).

Parmi les lecteurs interrogés, celles et ceux qui ont pu développer un goût pour la lecture de classiques - soit parce que leur parcours scolaire a accompagné leur ascension sociale, soit parce qu'ils étaient déjà suffisamment familiers avec la lecture pour s'approprier facilement les enseignements de français au collège et au lycée évoquent justement leur goût pour un regard analytique porté sur les œuvres, qui s'accompagne de compétences acquises au fil de leur trajectoire scolaire.

\footnotetext{
« Là on va s'intéresser à la vie de l'auteur, pourquoi cet auteur a écrit ce roman-là à une période de sa vie, est-ce que ça s'inscrit... Est-ce qu'il y a un fond d'histoire personnelle ou est-ce que c'est conflictuel par rapport à ce qui se passait à ce moment-là ? Donc oui ça m'a permis de contextualiser le livre... [...] Je pense que c'est intéressant parce que ça permet de positionner les lectures périphériques par rapport à ces classiques, ce qu'ils ont de différent, de mieux, de moins bien, peu importe » (Sabine, 36 ans, directrice marketing et commercial, bac +5 , père mécanicien, mère ouvrière en usine, grande lectrice).
}

Issue des classes populaires et ayant découvert le goût de la lecture par le biais de l'école, Sabine évoque ici l'influence que l'enseignement littéraire a eue sur sa pratique. Davantage que de lui faire découvrir une pratique culturelle, les enseignements délivrés à l'école lui ont permis de développer un rapport esthétique aux textes - qui se joue dans ses lectures actuelles -, qu'il s'agisse de classiques de la littérature ou pas : comparaison esthétique entre les auteurs, entre la littérature contemporaine et classique, recherche d'informations sur le contexte dans lequel les œuvres ont été écrites, etc. Pour ces lecteurs, l'école a joué deux rôles : l'accompagnement du développement de la lecture et l'apprentissage d'un rapport esthète à la lecture (Mauger \& Poliak, 1998 : 23).

On observe également chez des lecteurs moins dépendants vis-à-vis de l'école quant à la découverte de pratiques culturelles légitimes cette même transmission d'un rapport esthète à la littérature. Pierre, par exemple, a grandi dans un milieu familial aisé (cumulant capital culturel, scolaire et économique) et composé de lecteurs (ses parents, mais également ses sœurs et ses grands-parents); il a donc bénéficié d'une première socialisation favorable à la réussite scolaire ainsi qu'au développement de pratiques culturelles légitimes préalablement à l'école. L'école n'a joué aucun rôle dans sa découverte de la littérature ; en revanche, elle a accompagné la construction de son rapport aux textes littéraires dès le lycée, ce qui a été renforcé par son passage par une classe préparatoire, le distinguant du reste de sa famille malgré leur appartenance aux classes supérieures. Le rôle de l'école se lit particulièrement dans l'écart que Pierre évoque entre les conversations littéraires avec des membres de sa famille et celles avec ses amis (ayant une formation similaire) :

\footnotetext{
« Je pense que c'est assez bêtement lié au fait qu'on a fait les mêmes études, qu'on nous a appris à considérer comme légitime la même littérature, heu... pour la littérature contemporaine, on se renseigne assez par les mêmes canaux, je pense que déjà c'est assez déterminant, après on lit assez peu les mêmes choses au même moment. Je pense que c'est par homogénéité de goûts et de... de passif par rapport à ça qu'on lit les mêmes choses. Et puis vu qu'on a la même manière d'en parler, on est plus sensibles, je suis plus sensible à la manière qu'ils ont de me parler des œuvres qu'à d'autres manières où je percevrais moins l'intérêt que je pourrais y prendre ou le plaisir que j'aurais à lire ces œuvres en particulier » (Pierre, 24 ans, chef de publicité, bac +5 , père ingénieur, mère $\mathrm{RH}$, grand lecteur).
}

Ce rapport aux textes et à la lecture, qui s'appuie particulièrement à l'école sur un corpus d'œuvres classiques, est tout à fait transposable à la lecture d'œuvres contemporaines, puisées dans un répertoire d'œuvres légitimes, publiées chez des éditeurs relevant d'un champ de production restreinte (Bourdieu, 1971), saluées par la critique, et au sujet desquelles des conversations faisant appel à cet ensemble de compétences analytiques littéraires pourront se déployer. 


\section{L’hégémonie des classiques : une croyance sans pratique}

Les œuvres littéraires classiques dont la renommée est faite par l'école sont associées à l'idée d'une hiérarchie de la production littéraire, que les lecteurs une fois adultes vont généralement reprendre à leur compte, plaçant dans leurs discours la littérature classique au-dessus des autres productions. Ceci ne vient cependant pas assurer à ces œuvres qu'elles seront lues, puisqu'elles font également face à un certain nombre de réticences de la part des lecteurs.

\section{La croyance en la supériorité des œuvres classiques}

La majeure partie des lecteurs et lectrices interrogés ne remettent pas en cause, voire adhèrent pleinement à l'idée de la supériorité des œuvres classiques, qu'ils attribuent à leur traversée du temps. La beauté perçue dans ces œuvres est également au cœur des discours, notamment chez les lecteurs ayant hérité du goût pour la lecture de classiques ou qui ont réussi à franchir les barrières symboliques les en séparant 5 .

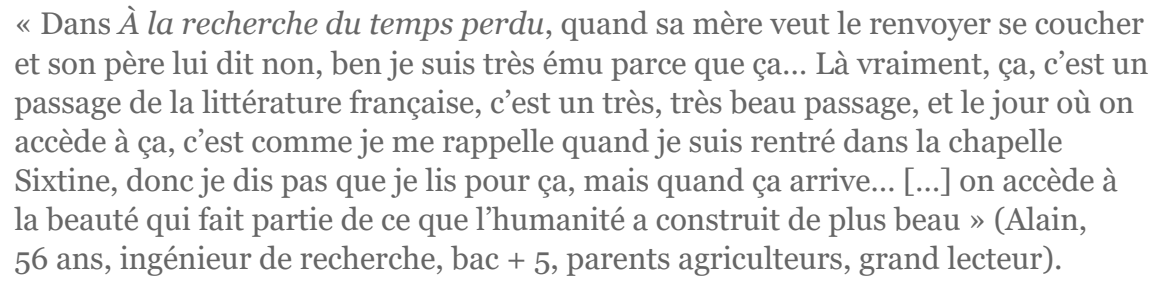

Nous pouvons corréler l'enthousiasme d'Alain au sujet de son expérience esthétique avec sa trajectoire ascendante et le sentiment d'un long chemin parcouru, la beauté des textes étant ici vécue sur le mode de la récompense. Mais on retrouve cet argument chez l'ensemble des lecteurs concernés par la lecture de classiques littéraires, et ce, bien qu'ils aient globalement conscience du caractère légitimant de ce type de lectures, de même que chez certains lecteurs et lectrices ne déclarant pourtant pas en lire. À l'inverse, certains se permettent plus de désinvolture à l'égard des œuvres consacrées, notamment lorsque leur milieu social, leur niveau d'étude et leur pratique de la lecture les autorisent à ce rapport plus détendu à la lecture de classiques.

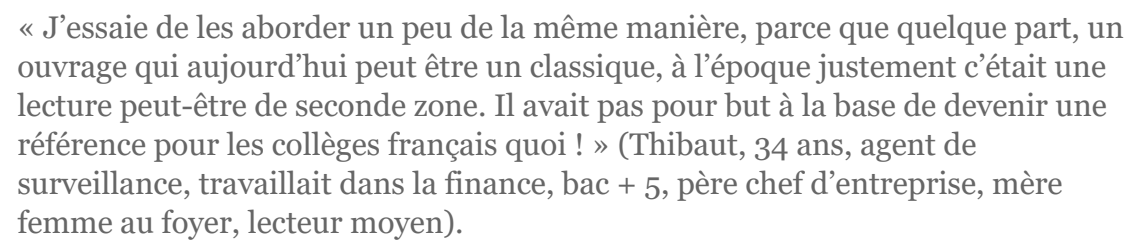

Héritier, diplômé du supérieur et présentant des modalités de la pratique distinctives, telles qu'un certain omnivorisme littéraire (Peterson \& Simkus, 1992) et un moindre attachement au livre comme objet, Thibaut peut se permettre un goût pour le classique qui ne passe pas par l'intimidation.

Chez le reste des lecteurs et lectrices, venant de milieux plus populaires, n'ayant pas eu de trajectoire ascendante, ne lisant pas de classiques, la valeur de ces œuvres semble aller de soi, sans faire autant l'objet de commentaires esthétiques.

\section{Des difficultés à se lancer dans la lecture de classiques}

L'ensemble des lecteurs interrogés reconnaissent - avec des degrés variables - la valeur de ces œuvres ainsi patrimonialisées, mais cette foi ne se transpose pas 
nécessairement dans des pratiques de lecture effectives. Les dernières données en date (Donnat, 2009) indiquent la faible place des classiques dans les préférences des Français, puisque $5 \%$ d'entre eux l'indiquent comme genre ${ }^{6}$ préféré (lorsque $18 \%$ d'entre eux indiquent préférer le genre policier, par exemple).

Figure 2. Genres littéraires lus selon l'âge des lecteurs (en \%).

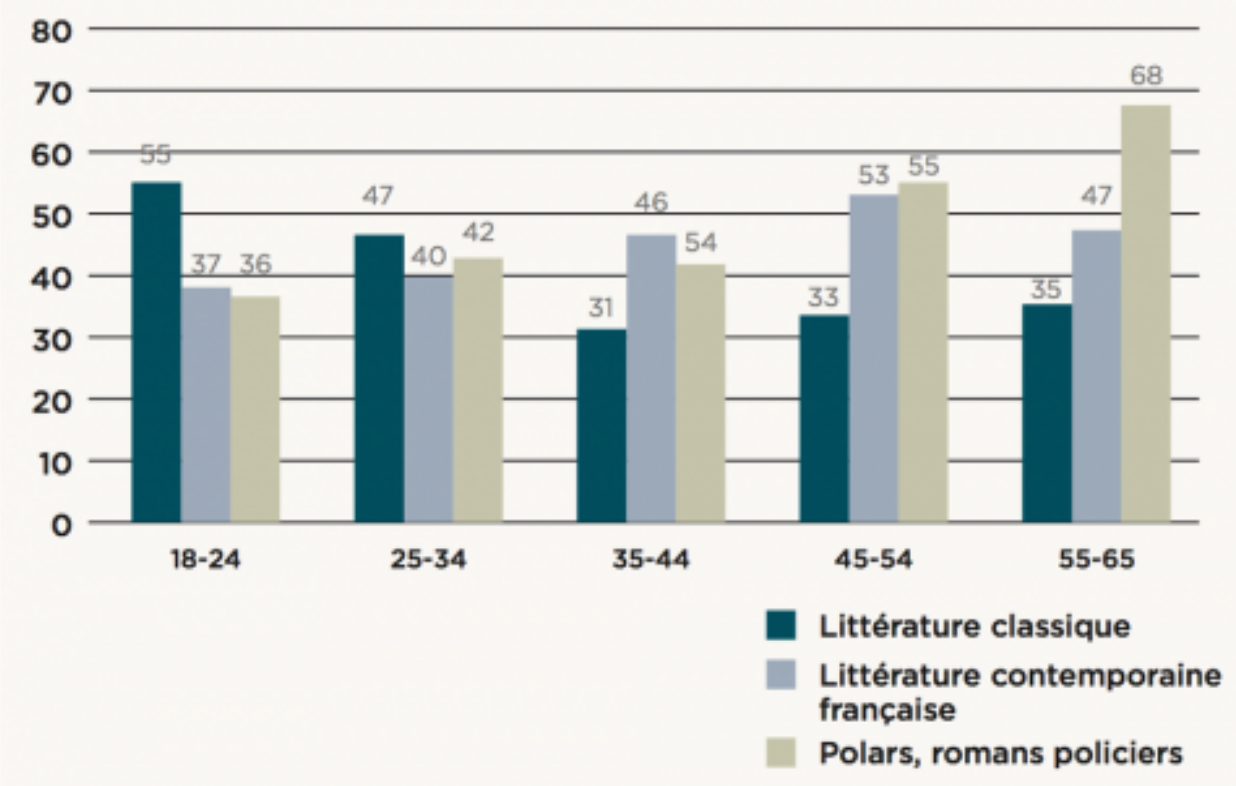

Dans notre enquête, les lecteurs interrogés âgés de moins de 34 ans déclarent plus fréquemment que les autres lire des classiques, ce qui peut s'expliquer par leur proximité plus ou moins récente avec le système scolaire, cette préférence diminuant au fur et à mesure qu'ils et elles s'en éloignent : $55 \%$ des lecteurs ayant entre 18 et 24 ans lisent des classiques contre $31 \%$ des $35-44$ ans et $35 \%$ des 55-65 ans.

Même lorsque les lecteurs interrogés appartiennent aux classes supérieures, qu'ils ont suivi des études supérieures et qu'ils lisent des classiques, ils évoquent de plus grandes difficultés à se tourner vers la lecture d'une œuvre classique plutôt que d'un roman plus récent.
« Mais y a tellement d'auteurs à découvrir, et je me dis que les Balzac et tout, ça a tellement été adapté en films, à la télé, là je peux dire que ces classiques-là, je me dis ça me suffit, parce qu'il y a tellement d'auteurs à découvrir, qui ont du talent, donc s'il faut se retaper du classique, c'est un peu du temps perdu, parce qu'on peut le voir autrement. [...] C'est aussi le fait de pas vouloir revenir en arrière. J'aime bien coller avec l'époque » (Pascale, 58 ans, employée administrative, BEP, père ouvrier, mère employée puis femme au foyer, faible lectrice).

Interrogés sur la comparaison entre les ouvrages classiques et contemporains, ils et elles se réfèrent fréquemment à cette représentation du classique comme plus sérieux, incitant moins au relâchement et à l'évasion (Mauger \& Poliak, 1998 : 4). Bien que le caractère obligatoire de ces lectures ait disparu, puisqu'elles ne se déploient plus dans le cadre de lectures imposées, des réticences au sujet du sérieux des œuvres classiques, des descriptions jugées longues qui les composent, du contexte passé dans lequel leur action se déroule, etc., détournent le lectorat adulte de leur lecture.

\footnotetext{
« Je suis restée assez fixée sur cette idée, c'est que le classique, c'est très descriptif, y a énormément de descriptions et ça, ça me soûle, j'aime bien quand ça va un peu vite, je suis pas portée sur le descriptif. Je sais que quand on parle de Proust et de ses madeleines, je sais qu'il y a je sais pas combien de pages dédiées à sa manière de parler des madeleines, patati patata, mais je sais pas si je vais vraiment accrocher. Je suis plus dans la littérature de l'actuel » (Khadija, 56 ans, maîtrenageuse, père inspecteur d'académie, mère femme au foyer, grande lectrice).
}

Les descriptions évoquées par les lecteurs semblent être un des traits constitutifs des œuvres classiques, caractérisant ce type d'œuvre en même temps qu'ils justifient sa mise à l'écart par des lecteurs craignant l'ennui. Cet extrait d'entretien montre bien 
comment la réputation d'une ouvre - conduisant à éviter sa lecture - est parfois pourtant fondée sur une moindre connaissance de son contenu, ce que l'évocation « des » madeleines de Proust, dont, selon la lectrice, il régalerait le lecteur pendant des pages et des pages, vient ici marquer.

Le sérieux associé à ces lectures découle également de la difficulté éprouvée vis-à-vis de leur lecture. Comme l'indiquent Baudelot et ses collègues (1999 : 47), « la longueur du livre est souvent le premier critère invoqué dans le jugement de goût porté sur un classique : "il est bien" s'il n'est pas trop long ", de la même manière que certains lecteurs vont se référer aux pages lues, ou au temps passé à lire, lorsqu'ils évoquent leur pratique de la lecture de ces œuvres spécifiques, rappelant l'effort mis en œuvre. La peur de se trouver face à un livre trop difficile d'accès se retrouve dans les discours d'un grand nombre de lecteurs qui trouvent là un frein à une pratique par laquelle certains d'entre eux sont malgré tout attirés, déplorant leur méconnaissance dans un mouvement simultané de fascination pour des œuvres sanctifiées et de déception vis-àvis de leur propre indignité culturelle.

\section{Des pratiques hétérogènes et socialement situées}

La grande majorité des lecteurs interrogés en entretien considère que la lecture de classiques revêt un caractère particulier, lié à une dimension relevant du domaine du sacré, qui la distingue de celle d'autres types de romans.

\footnotetext{
"Le classique c'est un peu un truc sacré, "un" classique, avec les warnings sur les côtés, t’as un peu plus la pression, l'écriture n'est pas la même, elle est moins fluide, alors que les romans, les polars ou autre c'est moins inaccessible, ça se passe soit dans une époque qu'on connait plus... ou alors finalement t'as surtout pas ce poids de l'histoire littéraire qui pèse sur toi quand tu veux lire le bouquin » (Marie, 35 ans, agent de communication dans le culturel, bac +5 , père vendeur, mère responsable de rayon dans la grande distribution, grande lectrice).
}

Se lancer dans la lecture de telles œuvres ne se fait pas avec la même facilité, et si une majorité de lecteurs affirment l'importance de lire des classiques, cette opinion ne se transforme pas en des pratiques effectives. Un ensemble de discours en demi-teinte au sujet de ce type d'œuvres a été relevé au fil des entretiens, pointant les difficultés des lecteurs à les lire, comme nous l'avons vu plus haut. Mais tous les lecteurs interrogés (à une exception près) s'accordent sur le statut supérieur des classiques, voire même sur la nécessité de les lire. À ce titre, on peut parler d'une croyance sans pratique (Legon, 2014) ; notre propos est ici que parmi les nombreux lecteurs et lectrices ayant développé une croyance en la valeur des œuvres patrimonialisées - et que ces derniers aient développé ou non un goût pour cette catégorie d'œuvres -, tous n'en ont pas pour autant développé une pratique de lecture.

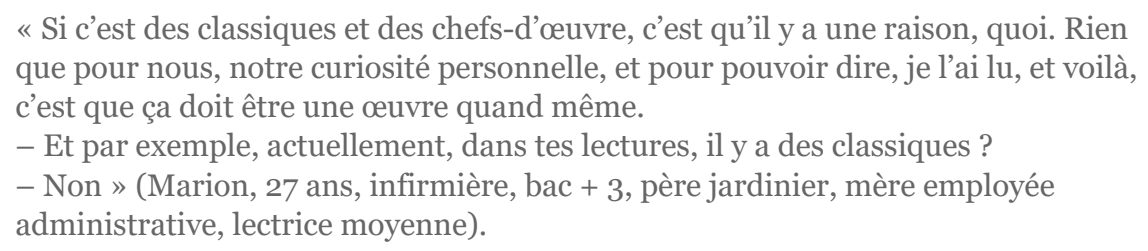

Une tension inhérente à la lecture de classiques se situe entre la nécessité évoquée par les lecteurs de lire des œuvres dont ils ne questionnent pas la valeur et celle de lire pour se divertir. C'est ici que réside la justification de ce paradoxe, ainsi que celle des écarts observables à ce sujet : si la lecture de divertissement est très répandue, quel que soit le milieu social ou le capital culturel des lecteurs, le rapport aux classiques diffère en revanche selon ces mêmes variables. C'est la capacité à avoir une lecture divertissante d'une œuvre classique - à associer classiques et évasion - qui explique chez certains le maintien ou le développement de la lecture de classiques. Ce sont donc les lecteurs et lectrices les plus dotés en capital culturel et scolaire - qu'ils viennent des classes supérieures ou qu'ils aient trouvé en l'école un lieu de réalisation personnelle et un outil de mobilité sociale - qui vont le plus fréquemment déclarer, parmi leurs 
lectures, des classiques : $45 \%$ des cadres, contre $31 \%$ des employés et $21 \%$ des lecteurs appartenant aux professions intermédiaires.

L'enseignement scolaire a donc cet effet contrasté d'encourager une partie du lectorat à les lire, tout en en dégoûtant une autre. Certains lecteurs - généralement issus des classes populaires et moyennes, souvent les plus réticents vis-à-vis de l'école et des lectures imposées - vont être amenés à développer un dégoût pour la lecture d'œuvres classiques (sans nécessairement en remettre en cause la valeur) qu'ils attribuent aux expériences scolaires passées ; la prescription scolaire a alors un effet repoussoir puisque l'expérience de lecture imposée d'une œuvre à l'adolescence vient en justifier tous les évitements une fois adulte. À l'inverse, lorsque les modalités propres à l'enseignement de la littérature sont acceptées par les lecteurs, ces derniers peuvent maintenir un rapport à la lecture teinté d'obligation, même si celle-ci est consentie. Plusieurs enquêtés évoquent notamment leurs lectures de classiques comme ils évoqueraient des devoirs de vacances, s'imposant parmi les lectures d'une année la lecture d'une ouvre classique imposante (Alain et Marie, précédemment cités, notamment), montrant là la bonne volonté culturelle de lecteurs en ascension sociale.

En somme, l'ensemble des lecteurs et lectrices interrogés semblent croire en la valeur de la littérature consacrée et diffusée par l'intermédiaire de l'école et des cours de français. Cette croyance a en revanche des effets d'attraction et de rejet différenciés, et se transpose donc différemment en pratiques. En matière de goût et de lecture effective d'œuvres littéraires classiques, plus on monte dans l'échelle sociale, plus l'appétence pour les classiques augmente, à mesure que l'intimidation diminue. Les pratiques des lecteurs en mobilité sociale sont très spécifiques et disent par ailleurs beaucoup du rôle de l'institution scolaire, la grande importance accordée par ces derniers à la culture légitime, et notamment au patrimoine littéraire, s'accompagnant d'un rapport à l'école particulier, puisqu'elle a joué un grand rôle tant dans leur trajectoire sociale que dans le développement de pratiques de lecture légitimes et valorisées par l'institution.

\section{Conclusion}

Nous avons montré que l'école n'était pas le lieu d'une médiation au livre et à la lecture efficace. S'adressant plus facilement à des lecteurs et lectrices présentant des caractéristiques sociales spécifiques (héritiers et transfuges), les romans classiques sur lesquels se porte l'enseignement du français au collège et au lycée ne rencontrent que difficilement leurs publics sur les bancs de l'école. Néanmoins, cette médiation n'est pas sans effets puisqu'elle participe à la patrimonialisation d'œuvres littéraires, par leur sélection dans les programmes et par leur analyse dans le cadre des cours de français. Cette patrimonialisation d'un corpus littéraire n'est à son tour pas sans effets sur les représentations associées à cette littérature (entre rejet, sacralisation et intimidation), qui s'accompagnent de pratiques de lectures ambivalentes (peu de lecteurs lisent des classiques mais tous reconnaissent la supériorité de cette littérature) et socialement situées. Que la littérature de classiques suscite le rejet, qu'elle soit intégrée à un ensemble de lectures sous la forme d'obligation, ou qu'elle corresponde à une réelle appétence, force est de constater que l'institution scolaire - par le biais des lectures imposées - parvient à asseoir la légitimité de cette littérature. En effet, lorsque les lecteurs mettent à distance les œuvres classiques, cela ne vient que rarement remettre en cause leur hégémonie, ces derniers associant fréquemment ce manque d'appétence à une incompétence de leur part plutôt qu'à leurs goûts ou à leur moindre valeur.

\section{Bibliographie}

Appadurai (Arjun). 1981. « The past as a scarce resource ». Man, 16, p. 201-219.

Baudelot (Christian), Cartier (Marie) \& Détrez (Christine). 1999. Et pourtant, ils lisent... Paris : Seuil (L'épreuve des faits).

Becker (Howard S.). 2010. Les Mondes de l'art. Traduit de l'anglais par Jeanne Bouniort. Paris : Flammarion (Champs arts) [1re édition en langue originale : 1982]. 
Bourdieu (Pierre). 1971. « Le marché des biens symboliques ». L’Année sociologique, 22, p. 49126.

Bourdieu (Pierre). 1979. La Distinction. Critique sociale du jugement. Paris : Minuit (Le sens commun).

Bourdieu (Pierre) \& Passeron (Jean-Claude). 1970. La Reproduction. Éléments pour une théorie du système d'enseignement. Paris : Minuit (Le sens commun).

Chartier (Anne-Marie) \& Hébrard (Jean). 2000. Discours sur la lecture (1880-200o). Paris : BpiCentre Pompidou / Fayard.

Donnat (Olivier). 2009. Les Pratiques culturelles des Français à l'ère numérique. Paris : La Découverte.

DOI : 10.3917/cule.095.0001

Dumazedier (Joffre) \& Hassenforder (Jean). 1959. " Le loisir et le livre ». Bulletin des bibliothèques de France (BBF), 6, p. 269-302

Éducation nationale. 2010. "Programme de l'enseignement commun de français en classe de seconde générale et technologique et en classe de première des séries générales, et programme de l'enseignement de littérature en classe de première littéraire ». Bulletin officiel de l'Éducation nationale, de la Jeunesse et des Sports, numéro spécial, 9, 30 septembre 2010, en ligne : https://www.education.gouv.fr/bo/2010/specialo9/mene1019760a.htm [consulté le 20 août 2021].

Eloy (Florence). 2015. Enseigner la musique au collège. Cultures juvéniles et culture scolaire. Paris : PUF (Éducation et société).

DOI : 10.3917/puf.flore.2015.01

Étienne (Jean-Marc). 2015. « Lectures d'œuvres de divertissement au collège ». Informations sociales, 190, p. 18-24.

DOI : 10.3917/inso.190.0018

Guillemot (André), Robine (Nicole) \& Escarpit (Robert). 1963. Le Livre et le conscrit. Bordeaux : Université de Bordeaux.

Guittet (Emmanuelle). 2018. Lecteurs et lectrices de romans face à la prescription littéraire : Une enquête sociologique sur les choix de lecture à l'ère numérique. Thèse de sociologie soutenue à l'Université Sorbonne Nouvelle - Paris 3.

Hersent (Jean-François). 200o. Sociologie de la lecture en France : État des lieux. Essai de synthèse des travaux de recherche menés en France. Paris : Direction du livre et de la lecture.

Kellner (Stéphanie). 2020. «Lire des classiques sur liseuse en bibliothèque municipale ». Biens symboliques / Symbolic Goods [en ligne], 7 : https://journals.openedition.org/bssg/482 [consulté le 20 août 2021] ; DOI : https://doi.org/10.4000/bssg.482.

DOI : $10.4000 /$ bssg.482

Lahire (Bernard). 1993a. Culture écrite et inégalités scolaires : Sociologie de l'échec scolaire à l'école primaire. Lyon : PUL.

Lahire (Bernard). 1993b. La Raison des plus faibles. Rapport au travail, écritures domestiques et lectures en milieux populaires. Villeneuve-d'Ascq : Presses universitaires du Septentrion.

Lahire (Bernard). 1995. Tableaux de famille. Heurs et malheurs scolaires en milieu populaire. Paris : Seuil.

Legon (Tomas). 2014. " Malentendus et désaccords sur le plaisir cinématographique. La réception de Lycéens et apprentis au cinéma par les jeunes rhônalpins ". Agora débats/jeunesses, 66, p. 47-60.

Lombardo (Philippe) \& Wolff (Loup). 2020. "Cinquante ans de pratiques culturelles en France ». Culture Études, 2020(2), p. 1-92.

DOI : 10.3917/cule.202.0001

Maridet (Morgane). 2016. La Khâgne, un nouveau chapitre : Élaborations et reconstructions du rapport à la lecture des étudiants en classe préparatoire littéraire. Thèse de sociologie soutenue à l'Université Sorbonne Nouvelle - Paris 3, en ligne : https://tel.archives-ouvertes.fr/tel01541454 [consulté le 20 août 2021].

Mauger (Gérard) \& Poliak (Claude). 1998. "Les usages sociaux de la lecture ». Actes de la recherche en sciences sociales, 123, p. 3-24.

DOI : 10.3917/arss.p1998.123n1.0003

Peterson (Richard A.) \& Simkus (Albert). 1992. « How musical tastes mark occupational status groups ", p. 152-186 in Cultivating Differences. Symbolic Boundaries and the Making of Inequality / sous la direction de Michèle Lamont et Marcel Fournier. Chicago : The University of Chicago Press.

Poulot (Dominique). 2006. Une histoire du patrimoine en Occident : XVIII ${ }^{e-X X I^{e}}$ siècle. Paris : PUF (Le nœud gordien).

Poulot (Dominique). 2011. "Le temps des musées et le temps du patrimoine ». Hermès, La Revue, 61, p. 23-29.

DOI : 10.3917/herm.061.0021 
Rabot (Cécile). 2012. « Le rapport des bibliothécaires de lecture publique aux auteurs ». Sociologie, 3(4), p. 359-376.

DOI : 10.3917/socio.034.0359

Renard (Fanny). 2011. Les Lycéens et la lecture. Entre habitudes et sollicitations. Rennes : PUR (Paideia).

Schön (Erich). 1993. " La "fabrication" du lecteur », p. 17-44 in Identité, lecture, écriture / sous la direction de Martine Chaudron et François de Singly. Paris : Centre Georges-Pompidou (Études et recherche).

Singly (François de). 1989. Lire à 12 ans : Une enquête sur les lectures des adolescents. Observatoire France Loisirs de la lecture. Paris : Nathan.

Singly (François de). 1993. "Le livre et la construction de l'identité », p. 131-152 in Identité, lecture, écriture / sous la direction de Martine Chaudron et François de Singly. Paris : Centre Georges-Pompidou (Études et recherche).

Viala (Alain). 1992. "Qu'est-ce qu'un classique ? ». Bulletin des bibliothèques de France (BBF), 1, p. 6-15.

DOI : 10.3406/licla.1993.1737

Viala (Alain). 1993. «Qu'est-ce qu'un classique ? ». Littératures classiques, 19, p. 11-31.

DOI : 10.3406/licla.1993.1737

\section{Notes}

1 Qui s'appuient sur une enquête de l'IFOP menée en 1955 et une enquête du Syndicat national de l'édition menée en 1960 (Hersent, 2000).

2 Parmi ces bienfaits supposés, la lecture favoriserait l'acquisition du langage, la réussite scolaire, aurait une influence sur la maîtrise de compétences scripturales, développerait l'imaginaire, etc.

3 Les questionnaires ont été administrés via des canaux diversifiés : devant des librairies et des bibliothèques parisiennes, au Salon du livre de Paris, sur des blogs et forums en ligne, ainsi qu'au sein de réseaux personnels où ils ont été diffusés par effet boule de neige ; les entretiens ont été menés avec des répondants du questionnaire.

4 Selon les catégories mobilisées dans les enquêtes sur les «pratiques culturelles des Français » du Département des études, de la prospective et des statistiques (DEPS) du ministère de la Culture : grands lecteurs (lisant plus de 20 romans par an), lecteurs moyens (entre 10 et 19) et faibles lecteurs (entre 1 et 9).

5 Quelle que soit la trajectoire des enquêtés, il arrive également que l'évocation de la beauté des textes vise à justifier un dédain pour des genres littéraires plus minimes.

6 Le classique est ici mobilisé comme un « genre », dans la lignée des enquêtes sociologiques sur les pratiques de lecture ; nous ne considérons pas qu'il s'agit d'un genre littéraire en soi, mais plutôt d'une catégorie qui fait sens pour les lecteurs et qu'il nous permet de nous inscrire dans le champ des études portant sur cette question.

7 De la même manière qu'on peut lire des classiques sans en avoir le goût, par adhésion à la croyance et par effet de légitimité.

\section{Table des illustrations}

\begin{tabular}{|c|c|c|}
\hline \multirow{2}{*}{$\mathbf{I} \mid \mathbf{E}$} & Titre & $\begin{array}{l}\text { Figure 1. Intérêt pour les lectures imposées en fonction de la fréquence } \\
\text { de lecture de l'entourage familial. }\end{array}$ \\
\hline & UيL & $\begin{array}{l}\text { http://journals.openedition.org/culturemusees/docannexe/image/6712/img- } \\
\text { 1.png }\end{array}$ \\
\hline & Fichier & image/png, 32k \\
\hline & Titre & Figure 2. Genres littéraires lus selon l'âge des lecteurs (en \%). \\
\hline 1 & UيL & $\begin{array}{l}\text { http://journals.openedition.org/culturemusees/docannexe/image/6712/img- } \\
\text { 2.png }\end{array}$ \\
\hline & & image/png, 32 \\
\hline
\end{tabular}

\section{Pour citer cet article}



lecture cède le pas à la valorisation du patrimoine littéraire », Culture \& Musées [En ligne], 38 | 2021, mis en ligne le 10 novembre 2021, consulté le 08 décembre 2021. URL : http://journals.openedition.org/culturemusees/6712 ; DOI : https://doi.org/10.4000/culturemusees.6712

\section{Auteur}

\section{Emmanuelle Guittet}

LabEx ICCA (Industries culturelles et création artistique), Cerlis

Emmanuelle Guittet est chercheuse postdoctorale au LabEx ICCA, en partenariat avec le Deps (ministère de la Culture) et associée au Cerlis (Centre de recherche sur les liens sociaux). Elle a soutenu en 2018, à l'Université Sorbonne Nouvelle - Paris 3 (Cerlis), une thèse de doctorat qui portait sur la prescription littéraire en régime numérique et son influence sur les pratiques de lecture de romans.

Ses travaux s'inscrivent dans une sociologie de la culture, des loisirs et du numérique et portent plus spécifiquement sur les pratiques de lecture, la prescription littéraire, les pratiques culturelles et de loisirs des adolescents, le rôle joué par le numérique dans les pratiques culturelles et de loisir, les loisirs créatifs et pratiques en amateur, la recommandation en ligne, les activités menées sur les réseaux et plateformes socionumériques.

Récemment, elle a publié « "Moi, il me faut du papier." Analyse d'une difficile et inégale conversion des lecteurs de romans au numérique " (Biens symboliques, $n^{\circ} 7,2020$ ) et « De l'évaluation à la critique amateur en ligne. Vers un renouveau des pratiques de recommandation ? » (Terminal, n¹28, 2020).

Courriel : emmanuelle.guittet[at]gmail.com

\section{Droits d'auteur}

Culture \& Musées 